\title{
Lidocaine-prilocaine cream was more effective than placebo for pain during circumcision
}

\author{
Taddio A, Stevens B, Craig K, et al. Efficacy and safety of lidocaine-prilocaine cream for pain during circumcision. N Engl J Med \\ 1997 Apr 24;336:1197-201.
}

\section{Objective}

To determine the efficacy and safety of lidocaine-prilocaine cream for reducing pain during circumcision in newborns.

\section{Design}

Randomised, double blind, placebo controlled trial with 24 hour follow up.

\section{Setting}

A teaching hospital in Ontario, Canada.

\section{Patients}

68 healthy white newborn babies (mean age 1.4 days) $\geqslant 37$ weeks gestation and birth weight $\geqslant 2500 \mathrm{~g}$ undergoing circumcision. Infants with jaundice or methaemoglobinaemia, and those receiving methaemoglobin inducing, analgesic, or sedative drugs were excluded. 8 infants $(12 \%)$ in the treatment group were excluded from the efficacy analysis because they were not treated under blind conditions.

\section{Intervention}

The infants were assigned to receive $5 \%$ lidocaine-prilocaine cream (1:1 mixture of lidocaine and prilocaine emulsified in water) $(n=38)$ or placebo cream containing coconut oil $(n=30)$. $1 \mathrm{ml}$ of cream was drawn into a syringe. One third of the dose was applied to the lower abdomen, and after extending the penis upward and pressing it against the abdomen, the remainder was applied to a dressing placed over the penis. The cream surrounded the penis for $60-80$ minutes, after which it was wiped off.

\section{Main outcome measures}

Pain indicated by a summary score of infant facial activity during the procedure, duration of crying, heart rate, blood pressure, and blood methaemoglobin.

\section{Main results}

During the surgery, the infants in the treatment group had lower facial activity scores $(\mathrm{p}=0.01)$, spent less time crying $(\mathrm{p}<0.001)$, and had a smaller overall increase in heart rate $(p=0.007)$ compared with the placebo group. The groups did not differ for blood pressure. None of the infants had clinical signs of methaemoglobinaemia.

\section{Conclusion}

Lidocaine-prilocaine cream was more effective than placebo for reducing pain (as indicated by facial activity and time spent crying) in newborn babies during circumcision, and had no adverse effects.

Sources of funding: Astra Pharma Inc, Canada, and the Medical Research Council of Canada Pharmaceutical Manufacturers Association.

For article reprint:Dr G Koren, Department of Pediatrics, Hospital for Sick Children, 555 University Avenue, Toronto, Ontario, M5G 1X8, Canada. Fax +1 4168137562

\section{Commentary}

The need to consider adequate analgesia for painful procedures on newborn babies is important to neonatal clinicians and parents. As such, the reporting of this study by Taddio $e t$ al is timely. In neonatal research, a major problem is the selection of an appropriate assessment tool that is sufficiently sensitive, valid, and reliable to measure pain response in newborn babies. In this study, the recording of facial expressions and physiological measures provided clinically relevant and appropriate indicators of pain experienced by newborn babies during the procedure.

A major strength of this study rests in blinding clinicians and assessors to the allocated treatment. This aimed to minimise bias in allocation to treatment or control groups, and subsequent pain assessment. The report, however, lacks detail as to the precise blinding process except that the creams were prepared and packaged by the sponsoring company. For example, no information was given regarding the steps taken to minimise risks of the blinding code being broken and so compromising the study results. In addition, no specific detail was provided regarding the precise method of random allocation to the 2 groups, which would enable the reader to determine whether all eligible newborns had an equal chance of allocation to the treatments under review. The withdrawal of 8 infants before data analysis also raises questions about selection bias. These limitations in reporting need to be considered.
In reaching their conclusion that the analgesic cream was more effective than the inert cream, the authors acknowledge that newborns in the experimental group still experienced pain during the procedure. Thus, the question is not so much whether some analgesia is better than no analgesia, but which is the most effective analgesic agent to use before painful procedures. Clinicians now need evidence based on a comparison of alternative analgesic agents to determine the most effective means of offering pain relief to newborn babies.

Jennifer Sleep, SRN, SCM, MTD

Professor of Nursing and Midwifery Research Thames Valley University London, UK 